\title{
A Simple Proof of a Theorem by Harris*
}

\author{
Guilherme Carmona \\ Universidade Nova de Lisboa
}

February 26, 2003

\begin{abstract}
We present a simple proof of existence of subgame perfect equilibria in games with perfect information.
\end{abstract}

\section{Introduction}

This note presents a simple proof of a theorem by Harris [4] on the existence of subgame perfect equilibria in games of perfect information. More generally, it illustrates a method for establishing existence of equilibria in such games, and allows for a new characterization of equilibrium outcomes that provides an algorithm for their computation.

The method we present consists of approximating the payoff function of each player by a sequence of simple functions, in a way that is standard in measure and integration theory (see, for example, Wheeden and Zygmund [5]). The sequence of approximating payoff functions for each player, in addition of being a sequence of simple functions, satisfies two other important properties: first, it converges uniformly to the payoff function of that player in the original game; and second, the approximation is such that outcomes that are dominated according to the some payoff function in the approximating sequence are also dominated (by the same outcome) according to the original payoff function, and to all the subsequent payoff functions in the sequence.

\footnotetext{
${ }^{*}$ I wish to thank Kemal Badur, and Jan Werner for very helpful comments. Financial support from the Subprograma Ciência e Tecnologia do $2^{\circ}$ Quadro Comunitário de Apoio is gratefully acknowledged.
} 
The above approximation of each player's payoff function induces a sequence of games that differ from the original game only on the payoff function. For this sequence of games, it is easy to establish that each of them has a nonempty, compact set of equilibrium outcomes, and that the sequence of those sets shrinks to the set of equilibrium outcomes of the original game - thus, the original game has a nonempty, compact set of equilibrium outcome. Furthermore, the set of equilibrium outcomes of the original game can be computed by intersecting the corresponding sets of the approximating games.

\section{Games of Perfect Information}

In order to present our argument in the simplest possible way, we will consider only the case of games of perfect information with two players. It should be noted that nothing in our argument depends on this assumption, and our proof could be easily extended to the case of an arbitrary finite number of players. Finally, using a "truncation argument" similar to the one used by Harris [4, section 4.4, page 624] (see also Börgers [1]), one could extent our result to the case of a denumerable number of players.

A game of perfect information (with two players) is

$$
G=\left\langle S_{i}, A_{2}, P_{i}\right\rangle_{i=1,2},
$$

where (1) $S_{i}$ is a nonempty set, for $i=1,2,(2) A_{2}$ is a nonempty valued correspondence from $S_{1}$ into $S_{2}$, and (3), $P_{i}: H \rightarrow \mathbb{R}$, for $i=1$, 2, where $H=\operatorname{graph}\left(A_{2}\right)$.

When player 2 moves at stage 2 , it is with perfect information on $s_{1}$. Hence the set of strategies of player $i, i=1,2$, is $F_{1}=S_{1}$ and

$$
F_{2}=\left\{f_{2}: S_{1} \rightarrow S_{2} \mid f_{t}\left(s_{1}\right) \in A_{2}\left(s_{1}\right)\right\} .
$$

A strategy vector $\left(f_{1}, f_{2}\right)$ is a subgame perfect equilibrium of $G$ if for all $s_{1} \in S_{1}$,

$$
f_{2}\left(s_{1}\right) \text { maximizes } P_{2}\left(s_{1}, \cdot\right) \text { in } A_{2}\left(s_{1}\right) \text {, }
$$

and

$$
P_{1}\left(f_{1}, f_{2}\left(f_{1}\right)\right) \geq P_{1}\left(s_{1}, f_{2}\left(s_{1}\right)\right) .
$$


Given a game of perfect information $G$ the vector $\left(f_{1}, f_{2}\left(f_{1}\right)\right)$ in $H$ determined uniquely by $\left(f_{1}, f_{2}\right)$ is called an equilibrium path of $G .{ }^{1}$ In what follows we show that the existence of an equilibrium is equivalent to the existence of an equilibrium path. Therefore, we can show that a subgame perfect equilibrium exists by showing that an equilibrium path exists, which is a simpler task. The reason is that, under the assumption we shall use, the set of equilibrium paths is compact, while the set of equilibrium strategies typically is not (see Harris [3, proposition 4]).

Define a correspondence $E_{2}$ from $S_{1}$ into $S_{2}$ as follows:

$$
E_{2}\left(s_{1}\right)=\left\{y \in A_{2}\left(s_{1}\right): P_{2}\left(s_{1}, y\right) \geq U_{2}\left(s_{1}, s_{2}\right), \quad \text { for all } s_{2} \in A_{2}\left(s_{1}\right)\right\}
$$

this correspondence gives the equilibrium paths of the one-player game $\left\langle A_{2}\left(s_{1}\right), P_{2}\right\rangle$. The set

$$
\begin{array}{cl}
E_{1}=\left\{(x, y) \in H \quad: \quad y \in E_{2}(x) \quad \text { and for all } \quad s_{1} \in S_{1} \text { there exists } s_{2} \in E_{2}\left(s_{1}\right)\right. \\
\text { such that } \left.P_{1}(x, y) \geq P_{1}\left(s_{1}, s_{2}\right)\right\}
\end{array}
$$

is then easily seen to be the set of equilibrium paths of the game $G=$ $\left\langle S_{i}, A_{2}, P_{i}\right\rangle_{i=1,2}$ (i.e., in the notation of footnote $1, E_{1}=P$.)

Lemma 1 A game of perfect information has a subgame perfect equilibrium if and only if $E_{1} \neq \emptyset$ and $E_{2}\left(s_{1}\right) \neq \emptyset$, for all $s_{1} \in S_{1}$.

Proof. Necessity follows from the fact that if $\left(f_{1}, f_{2}\right)$ is a subgame perfect equilibrium then $\left(f_{1}, f_{2}\left(f_{1}\right)\right) \in E_{1}$ and $f_{2}\left(s_{1}\right) \in E_{2}\left(s_{1}\right)$, for all $s_{1} \in S_{1}$.

For sufficiency, let $(x, y) \in E_{1}$. Define player 1 strategy by $f_{1}=x$. For player 2, we define $f_{2}\left(f_{1}\right)=y$. For $s_{1} \neq f_{1}$, because $(x, y)=\left(f_{1}, f_{2}\left(f_{1}\right)\right) \in E_{1}$, it follows that there exists $s_{2} \in E_{2}\left(s_{1}\right)$ such that

$$
P_{1}\left(f_{1}, f_{2}\left(f_{1}\right)\right) \geq P_{1}\left(s_{1}, s_{2}\right)
$$

hence we define $f_{2}\left(s_{1}\right)=s_{2}$.

${ }^{1}$ More precisely, the set of equilibrium paths of a game of perfect information is $P=$ $\left\{(x, y) \in H\right.$ : there exists an subgame perfect equilibrium $\left(f_{1}, f_{2}\right)$ such that $f_{1}=x$ and $\left.f_{2}\left(f_{1}\right)=y\right\}$. 


\section{Approximation by Simple Functions and Harris' Theorem}

Let $G=\left\langle S_{i}, A_{2}, P_{i}\right\rangle_{i=1,2}$ be a game of perfect information, and assume that for all $i=1,2, P_{i}(H) \subset[0,1)$. For $i=1,2$ and $k \in \mathbb{N}$, let $P_{i}^{k}: H \rightarrow \mathbb{R}$ be defined by

$$
P_{i}^{k}(h)=\frac{j-1}{2^{k}} \quad \text { if } \quad \frac{j-1}{2^{k}} \leq P_{i}(h)<\frac{j}{2^{k}},
$$

for $j=1, \ldots, 2^{k}$. Define $G_{k}=\left\langle S_{i}, A_{2}, P_{i}^{k}\right\rangle_{i=1,2}$, for all $k \in \mathbb{N}$. We have that $\left\|P_{i}^{k}-P_{i}\right\|_{\infty} \leq \frac{1}{2^{k}}$, and also that $P_{i}^{k}$ is a simple function; this last fact allows us to solve the game $G_{k}$ by backwards induction, and thus show that it has a subgame perfect equilibrium. The following lemma summarizes this fact.

Lemma 2 For all $k \in \mathbb{N}, G_{k}$ has a subgame perfect equilibrium.

Proof. It follows immediately by backward induction:

Let $s_{1} \in S_{1}$. Since $P_{2}$ is simple, then $\left\{P_{2}\left(s_{1}, s_{2}\right): s_{2} \in A_{2}\left(s_{1}\right)\right\}$ is finite, and so there exists $s_{2}^{*}$ that maximizes $s_{2} \mapsto P_{2}\left(s_{1}, s_{2}\right)$ in $A\left(s_{1}\right)$. Thus, define $f_{2}\left(s_{1}\right)=s_{2}^{*}$. This defines an optimal strategy $f_{2}: S_{1} \rightarrow S_{2}$ for player 2 .

Similarly, since $P_{1}$ is simple, then $\left\{P_{1}\left(s_{1}, f_{2}\left(s_{1}\right)\right): s_{1} \in S_{1}\right\}$ is finite, and so there exists $s_{1}^{*}$ that maximizes $s_{1} \mapsto P_{1}\left(s_{1}, f_{2}\left(s_{1}\right)\right)$ in $S_{1}$. Thus, define $f_{1}=s_{1}^{*}$. This defines an optimal strategy $f_{1} \in S_{1}$ for player 1 . Clearly, $f=\left(f_{1}, f_{2}\right)$ is a subgame perfect equilibrium.

We will prove Harris' theorem by approximating a given game $G$ by a sequence of games $\left\{G_{k}\right\}_{k=1}^{\infty}$ as defined above. In fact, this will be a consequence of Theorem 1 below, which shows that the set of equilibrium outcomes of $G_{k}$ converges to the set of equilibrium outcomes of $G$.

Theorem 1 Let $G=\left\langle S_{i}, A_{2}, P_{i}\right\rangle_{i=1,2}$, be a game of perfect information.

Suppose that

1. for all $i=1,2, S_{i}$ is a compact topological space;

2. $H$ is a closed subset of $S_{1} \times S_{2}$;

3. for all $i=1,2, P_{i}$ is continuous;

4. $A_{2}: S_{1} \rightarrow \rightarrow S_{2}$ is lower hemicontinuous. 
Then, $E_{1}=\cap_{k=1}^{\infty} E_{1}^{k}=\cap_{k=1}^{\infty} \overline{E_{1}^{k}}$ and $E_{2}\left(s_{1}\right)=\cap_{k=1}^{\infty} E_{2}^{k}\left(s_{1}\right)=\cap_{k=1}^{\infty} \overline{E_{2}^{k}\left(s_{1}\right)}$, for all $s_{1} \in S_{1}$.

Proof. First note that because $H$ is compact and $P_{i}$ is continuous for $i=1,2$, there is no loss in generality by assuming that $P_{i}(H) \subset[0,1)$, for all $i=1,2$.

We show first that $E_{1} \subseteq \cap_{k=1}^{\infty} E_{1}^{k}$ and $E_{2}\left(s_{1}\right) \subseteq \cap_{k=1}^{\infty} E_{2}^{k}\left(s_{1}\right)$, for all $s_{1} \in S_{1}$. This follows from the fact that for all $i=1,2$ and $h, l \in H, P_{i}(h) \geq P_{i}(l)$ implies $P_{i}^{k}(h) \geq P_{i}^{k}(l)$.

We show next that $\cap_{k=1}^{\infty} \overline{E_{1}^{k}} \subseteq E_{1}$. Let $(x, y) \in \cap_{k=1}^{\infty} \overline{E_{1}^{k}}$. Then, for each $k \in \mathbb{N}$, there a net $\left\{\left(x_{j}^{k}, y_{j}^{k}\right)\right\}_{j \in J_{k}} \subseteq E_{1}^{k}$ converging to $(x, y)$.

First, we will show that $y \in E_{2}(x)$. Let $s_{2} \in A_{2}(x), k \in \mathbb{N}$, and $\left\{s_{j}\right\}_{j \in J_{k}}$ be such that $s_{j} \in A\left(x_{j}^{k}\right)$ for all $j$, and $s_{j} \rightarrow s_{2}$ (such net exist since $A$ is lower hemi-continuous). Since for all $j \in J_{k}$ we have that $y_{j}^{k} \in E_{2}^{k}\left(x_{j}^{k}\right)$, then

$$
P_{2}^{k}\left(x_{j}^{k}, y_{j}^{k}\right) \geq P_{2}^{k}\left(x_{j}^{k}, s_{j}\right)
$$

for all $j \in J_{k}$. Since $\left\|P_{2}^{k}-P_{2}\right\|_{\infty} \leq \frac{1}{2^{k}}$, we obtain

$$
P_{2}\left(x_{j}^{k}, y_{j}^{k}\right) \geq P_{2}\left(x_{j}^{k}, s_{j}\right)-\frac{2}{2^{k}}
$$

and so,

$$
P_{2}(x, y) \geq P_{2}\left(x, s_{2}\right)-\frac{2}{2^{k}},
$$

since $P_{2}$ is continuous. Finally, letting $k \rightarrow \infty$, it follows that

$$
P_{2}(x, y) \geq P_{2}\left(x, s_{2}\right)
$$

Hence, it follows that $y \in E_{2}(x)$.

We are left to show that for all $s_{1} \in S_{1}$ there exists $s_{2} \in E_{2}\left(s_{1}\right)$ such that $P_{1}(x, y) \geq P_{1}\left(s_{1}, s_{2}\right)$. Let $s_{1} \in S_{1}$ and $k \in \mathbb{N}$. For each $j \in J_{k}$, let $w_{j}^{k} \in E_{2}^{k}\left(s_{1}\right)$ be such that

$$
P_{1}\left(x_{j}^{k}, y_{j}^{k}\right) \geq P_{1}\left(s_{1}, w_{j}^{k}\right) .
$$

Since $\left\{w_{j}^{k}\right\}_{j \in J_{k}} \subseteq A_{2}\left(s_{1}\right)$, and $A_{2}\left(s_{1}\right)$ is compact, we may assume that $\left\{w_{j}^{k}\right\}_{j \in J_{k}}$ converges; let $w^{k}$ be such that $w_{j}^{k} \rightarrow w^{k}$. The sequence $\left\{w^{k}\right\}_{k \in \mathbb{N}}$ lies also on $A_{2}\left(s_{1}\right)$ and so we may again assume that it converges; let $w$ be 
such that $w^{k} \rightarrow w$. An argument parallel to the one used above establishes that

$$
P_{1}(x, y) \geq P_{1}\left(s_{1}, w\right) .
$$

Hence, it is enough to show that $w \in E_{2}\left(s_{1}\right)$, which can again be done with an argument similar to the one used to show that $y \in E_{2}(x)$.

Similarly, one can show that $\cap_{k=1}^{\infty} \overline{E_{2}^{k}\left(s_{1}\right)} \subseteq E_{2}\left(s_{1}\right)$, for all $s_{1} \in S_{1}$. This completes the proof.

Theorem 1 gives a characterization of subgame perfect equilibrium paths of $G$ in terms of the equilibrium paths of the approximating games, and provides an algorithm for their computation. Also, it follows immediately from Theorem 1 that $E_{1}$ and $E_{2}\left(s_{1}\right)$, for all $s_{1} \in S_{1}$, are compact. Also, Harris' Theorem follows easily from Lemma 2, and Theorem 1.

Theorem 2 (Harris) Let $G$ be a game of perfect information. Under the same assumptions of Theorem 1, G has a subgame perfect equilibrium.

Proof. For $k \in \mathbb{N}$, let $G_{k}$ be as defined above. By Lemma 2 it follows that $E_{2}^{k}\left(s_{1}\right)$ is nonempty, for all $s_{1} \in S_{1}$, and that $E_{1}^{k}$ is nonempty. Since, for all $k \in \mathbb{N}, i=1,2$ and $h, l \in H, P_{i}^{k+1}(h) \geq P_{i}^{k+1}(l)$ implies $P_{i}^{k}(h) \geq P_{i}^{k}(l)$, it follows that $E_{2}^{k+1}\left(s_{1}\right) \subseteq E_{2}^{k}\left(s_{1}\right)$, for all $s_{1} \in S_{1}$ and that $E_{1}^{k+1} \subseteq E_{1}^{k}$.

Claim 1 For all $s_{1} \in S_{1}, A_{2}\left(s_{1}\right)$ is closed.

Proof. Let $\left\{y_{j}\right\}_{j \in J}$ be a convergent net in $A_{2}\left(s_{1}\right)$ and let $y \in S_{2}$ be such that $y_{j} \rightarrow y$. Since $\left(s_{1}, y_{j}\right) \in H$ for all $j \in J$, and $H$ is closed, it follow that $\left(s_{1}, y\right) \in H$. Hence, $y \in A_{2}\left(s_{1}\right)$.

Since for all $s_{1} \in S_{1}, A_{2}\left(s_{1}\right)$ is closed it follows that $\overline{E_{2}^{k}\left(s_{1}\right)} \subseteq C_{2}\left(s_{1}\right)$, for all $k \in \mathbb{N}$; furthermore, for all $s_{1} \in S_{1}$ and $k \in \mathbb{N}, \overline{E_{2}^{k}\left(s_{1}\right)}$ is a nonempty, closed subset of a compact space and $\overline{E_{2}^{k+1}\left(s_{1}\right)} \subseteq \overline{E_{2}^{k}\left(s_{1}\right)}$. Hence, for all $s_{1} \in S_{1}, \cap_{k=1}^{\infty} \overline{E_{2}^{k}\left(s_{1}\right)}$ is a nonempty subset of $A_{2}\left(s_{1}\right)$ (see Kelley [2, theorem 1 , page 136]). Similarly, we can conclude that $\cap_{k=1}^{\infty} \overline{E_{1}^{k}}$ is a nonempty subset of $H$. Hence, it follows from Theorem 1 that $E_{2}\left(s_{1}\right)$ is nonempty, for all $s_{1} \in S_{1}$, and that $E_{1}$ is nonempty, which completes the proof.

Remark 1 Note that the above Theorem dispenses with the assumption used by Harris [4] that $S_{i}, i=1,2$, is Hausdorff. 


\section{References}

[1] Börgers, T., (1991), "Upper Hemicontinuity of the Correspondence of Subgame-Perfect Equilibrium Outcomes," Journal of Mathematical Economics, 20, 89-106.

[2] Kelley, J., (1955), General Topology, New York: Springer.

[3] Harris, C., (1985), "A Characterizarion of the Perfect Equilibria of Infinite Horizon Games," Journal of Economic Theory, 37, 99-125.

[4] Harris, C., (1985), "Existence and Characterization of Perfect Equilibrium in Games of Perfect Information," Econometrica, 53, 613-628.

[5] Wheeden, R. and A. Zygmund, (1977), Measure and Integral: an Introduction to Real Analysis, New York: Dekker. 